\title{
Proteomic identification of specific glycosyltransferases functionally implicated for the biosynthesis of a targeted glyco-epitope
}

\author{
Chi-Hung Lin ${ }^{1,2,3}$, Chia-Wei Lin ${ }^{4}$ and Kay-Hooi Khoo 1,3,4 \\ ${ }^{1}$ Chemical Biology and Molecular Biophysics Program, Taiwan International Graduate Program, \\ Academia Sinica, Taipei, Taiwan \\ ${ }^{2}$ Institute of Bioinformatics and Structural Biology, National Tsing-Hua University, Hsin-Chu, Taiwan \\ ${ }^{3}$ Institute of Biological Chemistry, Academia Sinica, Taipei, Taiwan \\ ${ }^{4}$ Institute of Biochemical Science, National Taiwan University, Taipei, Taiwan
}

\begin{abstract}
Functional glycomic and glycoproteomic analyses often entail correlating the mapped glycosylation pattern of a cell against the activities of specific glycosyltransferases it expresses. While the mRNA transcripts can be readily mapped, the expression of a functional glycosyltransferase at protein level has defied most current proteomic approaches. To enable identification of these low abundant Golgi residing membrane bound proteins, we have developed a novel semigel-based shotgun proteomic workflow incorporating subcellular fractionation and one-step affinity enrichment of the detergent solubilized Golgi preparation on resins derivatized with nucleotide diphosphates. Applying the strategy to a colonic adenocarcinoma, Colo205, which is known to aberrantly synthesize abundant fucosylated extended type 1 chain, we first validated that $\beta 3$ galactosyltransferase 5 ( $\beta 3 \mathrm{GalT5}$ ) is indeed the overexpressed $\beta 3 \mathrm{GalT}$. This and $\beta 4 \mathrm{GalT} 1$ are the two galactosyltrasferases which were positively identified by proteomic analysis of the eluted fractions from uridine diphosphate (UDP)-affinity column. Substituting UDP with a guanidine diphosphate (GDP)-affinity column and monitoring the eluted fractions for enriched $\alpha 3 / 4$-fucosyltransferase (FucT) activities, we then identified FucT3 and FucT6 as the two major $\alpha 3 / 4 \mathrm{FucTs}$ expressed in Colo205 at the protein level. Our proteomic analysis demonstrated that not all GDPutilizing glycosyltransferases bind and are retained similarly by the GDP-affinity column and that specific activity assay along with optimization of binding and elution conditions is critical for successful identification of a particular subset of the targeted glycosyltransferases. Only OFUT1, a protein $O$-FucT, was additionally identified to coelute with the $\alpha 3 / 4 \mathrm{FucT}$ activity, and not other GDP-fucose utilizing FucTs.
\end{abstract}

Received: July 19, 2007

Revised: November 7, 2007

Accepted: November 9, 2007

Keywords:

Affinity capture / Glycosyltransferases / Subcellular fractionation

Correspondence: Dr. Kay-Hooi Khoo, Institute of Biological Chemistry, Academia Sinica, 128, Academia Rd Sec 2, Nankang Taipei 11529, Taiwan - Republic of China

E-mail: kkhoo@gate.sinica.edu.tw

Fax: +886-2-27889759

Abbreviations: FucT, fucosyltransferase; GalT, galactosyltransferase; GDP, guanidine diphosphate; Le ${ }^{b}$, Lewis b, Fuc $\alpha 1-2$ Gal $\beta 1-$ (Fuc $\alpha 1-3) 4$ GlcNAc $\beta$-; (s)Lex , (Sialyl) Lewis $x$, (sialyl)Gal $\beta 1$-(Fuc $\alpha 1$ 3) 4 GIcNAc $\beta-$; LNnT, neolactotetraose, Gal $\beta 1-4$ GIcNAc $\beta 1-3 G a l \beta 1-$ 3Glc; PA, 2-aminopyridine; TX-100, Triton X-100; UDP, uridine diphosphate

\section{Introduction}

Glycan chains are known to modulate many biological functions such as signaling, immune response, and development, in part by mediating cell-cell and protein-protein interaction through terminal glyco-epitopes. The developmental stage and tissue specific expression of a particular glyco-epitope is mostly effected through concerted actions of glycosyltransferases residing in the Golgi apparatus. The most direct biochemical approach to understand and manipulate the functions of glycosylation is therefore to 
determine the regulated expression of each of the implicated endogenous glycosyltransferases, followed by the studies of substrate specificities and kinetics of individually purified enzymes. A major difficulty, however, is that these enzymes are often unstable and very low in abundance [1]. Purification of glycosyltransferase usually requires large quantity of animal tissue and multiple chromatography steps. Moreover, since most of these enzymes are ER and Golgi resident type II membrane proteins [2], the requisite use of detergent for solubilization and prevention of aggregation often complicates the purification procedure.

The first mammalian glycosyltransferase gene was cloned by using the partial sequence of purified enzyme [3]. The progress of gene cloning was slow then because purification is difficult and time consuming. In the early $90 \mathrm{~s}$, expression cloning and PCR cloning were extensively used to identify novel glycosyltransferase genes. This led to the current picture of mammalian glycosyltransferase families, each with its distinctive motifs, as well as common characteristics. More recently, in silico analysis of glycosyltransferase gene sequences together with advances in human genome projects has brought forth a comprehensive identification and cloning of human glycosyltransferase genes including those of unknown specificities and/or functions $[4,5]$. This development provides a relatively easy way to map the expression of mRNA transcript, which is conveniently taken as an indicator of the expressed enzyme activity.

A fundamental scientific rationale for proteomics is that expression of a gene transcript is often a good indicator but not equivalent to functional protein expression or activity. There are growing examples, which collectively show that the mRNA transcript level may not accurately reflect the true expression level of a functional protein [6] that is subjected to further translational and post-translational regulation. In the case of glycosyltransferases, the in vivo activity and specificity of the enzymes are determined not only at the transcription level but also by their subcellular localization, proteolytic processing, dimerization/oligomerization, and PTMs [7, 8], which therefore necessitate the identification and biochemical studies of endogenous enzymes. Furthermore, while the overall activity of a particular class of glycosyltransferases can be readily demonstrated by in vitro assay of cell lysates using appropriate acceptor, it remains difficult to directly attribute the observed activities to any one of the many family members without a facile way to identify the expressed enzymes at protein level.

Recent technical advances in MS-based proteomics have made it possible to identify proteins with greater ease and sensitivity than previously possible. Subcellular organellar proteomic studies have further provided many detailed mapping of the constituent proteins of various organelles [9]. Among these, large scale Golgi proteomic studies using rat liver as source material have led to the identification of an impressive list of Golgi proteins including many novel ones [10-13] but only a limited number of glycosyltransferases [12, 13]. The most commonly found glycosyltransferases are polypeptide $N$-acetylgalactosaminyltransferases for initiation of mucin type $\mathrm{O}$-glycosylation, $\mathrm{N}$-acetylglucosaminyltransferases (GnTs) for conversion of high mannose type $\mathrm{N}$-glycans to complex type, the ubiquitous $\beta 1,4$-galactosyltransferases (GalTs), and some of the sialyltransferases, which collectively represent only a tiny fraction of all glycosyltransferases implicated to be functionally active for a glycome under investigation. The only fucosyltransferase (FucT) identified in large scale Golgi proteomics is FucT8 [13], which catalyzes core fucosylation of $N$-glycan. Furthermore, very few Golgi proteomic studies actually used cultured human cells [14] and thus far no proteomic identification of human glycosyltransferases from this sample source has ever been reported. These suggest that specifically tailored isolation and enrichment step would be required to identify glycosyltransferases by MS-based proteomic approach.

We have initiated studies along this line to develop analytical strategy which would allow proteomic identification of low abundant glycosyltransferases inferred to be responsible for the synthesis of glyco-epitopes characteristic of a cell line, as defined by initial glycomic mapping. Colo205, a colonic adenocarcinoma cell line, was reported to have high level of fucosylation presented as terminal Lewis $\mathrm{a}\left(\mathrm{Le}^{\mathrm{a}}\right)$ and the tumor specific dimeric $\mathrm{Le}^{\mathrm{b} / \mathrm{a}}-\mathrm{Le}^{\mathrm{a}}$ glycotopes on their lacto-series glycosphingolipids [15]. The preference for a backbone sequence based on type 1 chain, -Gal $\beta 1-3 G l c N A c \beta 1$-, instead of the more common type 2 chain, -Gal $\beta 1-4$ GlcNAc $\beta 1-$, has been attributed to abnormally high activity of $\beta 3 \mathrm{GalT}$ [16]. Using this as our model system, we show here that proteomic identification of glycosyltransferases from microsomal or Golgi preparation of cell culture is possible after one-step enrichment with uridine diphosphate (UDP)/guanidine diphosphate (GDP)-affinity column, coupled with careful monitoring of the targeted enzyme activity. The latter is critical and required since not all glycosyltransferases that utilize UDP- or GDP-based nucleotide sugar diphosphates as donors would bind equally well to the respective affinity column and eluted similarly. We identified $\beta 3 \mathrm{GalT5}$ as the predominantly expressed $\beta 3 \mathrm{GalT}$, along with FucT3 and FucT6 which contributed to the expressed $\alpha 3 / 4$ FucT activities.

\section{Materials and methods}

\subsection{Materials}

All chemicals were purchased from Sigma unless otherwise noted. GDP-affinity resin, UDP-affinity resins, and pyridylaminated sugar chains 2-aminopyridine (PA-sugar) were purchased from Calbiochem. 


\subsection{Glycosyltransferase assay}

Glycosyltransferase activity assays were based on detecting the enzymatic products by HPLC, using PA-glycans as acceptors. For $\beta 3 \mathrm{GalT}$ and $\beta 4 \mathrm{GalT}$ activities, GlcNAc $\beta 1$-LacPA was used as acceptor substrate (where Lac stands for Gal $\beta 1-4 \mathrm{Glc}$ ). For activity assay, $5 \mu \mathrm{L}$ of the enzyme source was added to give a final volume of $20 \mu \mathrm{L}$ reaction solution containing $50 \mathrm{mM}$ HEPES buffer, $\mathrm{pH} 7.0$, containing $1 \%$ Triton X-100 (TX-100), 20 mM MnCl $2,3.3$ mM UDP-Gal, and $5 \mu \mathrm{M}$ PA-glycan acceptors. Reaction was conducted at $37^{\circ} \mathrm{C}$ for $4 \mathrm{~h}$. For FucT activity assay, $10 \mathrm{mM}$ GDP-fucose and $10 \mu \mathrm{M}$ neolactotetraose (LNnT)-PA were used as donor and acceptor substrates, respectively. After boiling at $100^{\circ} \mathrm{C}$ for $2 \mathrm{~min}$ and centrifugation at $13000 \mathrm{rpm}$ for $3 \mathrm{~min}$, supernatant from the reaction mixture was applied to a TSK-gel ODS- $80^{\text {TM }}$ column $(4.6 \times 250 \mathrm{~mm}$; Tosoh, Japan $)$ fitted on an Agilent HP1100 HPLC system coupled with an HP1100 fluorescence detector. Products and nonreacted acceptors were eluted isocratically with $20 \mathrm{mM}$ ammonia acetate at $\mathrm{pH} 4.0$ and identified by elution time in reference to authentic PAglycan standards. Further confirmation of the structures was afforded by MALDI-MS and MS/MS analysis of the collected peaks as permethyl derivatives, using an MALDI-TOF/TOF mass spectrometer (4700 Proteomics Analyzer, Applied Biosystems, Framingham, MA), as described in ref. [17].

\subsection{Cell culture and preparation of microsome and Golgi membrane fractions}

Colo205 cells were cultured in RPMI 1640 medium containing $10 \%$ FCS in $75 \mathrm{~cm}^{2}$ culture flasks. After harvest, cells $\left(\sim 1 \times 10^{8}\right)$ were washed three times in homogenization HES buffer (20 mM HEPES, pH 7.5, containing 1 mM EDTA and $0.25 \mathrm{M}$ sucrose) and cell lysate was prepared by 20 strokes of a Dounce device. Cell debris was removed by centrifugation at $2000 \times \mathrm{g}$ for $10 \mathrm{~min}$. Postnuclear fraction was then subjected to ultrancentrifugation at $105000 \times \mathrm{g}$ in an SW $41 \mathrm{Ti}$ rotor for $16 \mathrm{~h}$. The resulting pellet was collected as microsome fraction for activity assay and proteomic analysis. To obtain the Golgi membrane, the postnuclear fraction was subjected to ultracentrifugation at $105000 \times \mathrm{g}$ for $16 \mathrm{~h}$ in an SW 41 Ti rotor with step sucrose gradient from 15 to $55 \%$. Aliquots of $0.5 \mathrm{~mL}$ were taken from the top of the centrifugation tube. The collected fractions were then assayed for $\beta$ GalT activity to define the Golgi membrane enriched fractions, which were pooled, diluted by HES buffer, and pelleted by $105000 \times \mathrm{g}$ for $1 \mathrm{~h}$.

\subsection{Extraction of target glycosyltransferases and affinity chromatography}

Enriched microsome or Golgi membrane pellet was resuspended and extracted by $50 \mathrm{mM}$ HEPES buffer, pH 7.0, containing $20 \mathrm{mM} \mathrm{MnCl}_{2}, 150 \mathrm{mM} \mathrm{NaCl}, 1 \mathrm{mM}$ DTT, $20 \%$ glycerol, and $1 \% \mathrm{TX}-100$, for $16 \mathrm{~h}$. Insoluble material was removed by ultracentrifugation at $105000 \times \mathrm{g}$ for $1 \mathrm{~h}$ and the supernatant was collected as TX-100 extraction for direct activity assays or further affinity enrichment. For GalT, $1.5 \mathrm{~mL}$ UDP-affinity $(10 \mu \mathrm{mol} / \mathrm{mL})$ resin was packed and equilibrated with binding buffer (50 mM HEPES, pH 7.0, $20 \mathrm{mM}$ $\mathrm{MnCl}_{2}, 150 \mathrm{mM} \mathrm{NaCl}, 1 \mathrm{mM}$ DTT, 20\% glycerol, 1\% TX100). For FucT, $1.5 \mathrm{~mL}$ GDP-affinity $(10 \mu \mathrm{mol} / \mathrm{mL})$ resin was packed and equilibrated with the same binding buffer except for the use of $20 \mathrm{mM} \mathrm{MgCl}$, instead of $\mathrm{MnCl}_{2}$. TX-100 extraction was loaded into column and washed by $3 \times$ volume of the binding buffer. Bound fraction was eluted sequentially by $0.5 \mathrm{M} \mathrm{NaCl}, 20 \mathrm{mM}$ EDTA, and $2 \mathrm{mM}$ UDP for GalT, or $0.5 \mathrm{M} \mathrm{NaCl}$ and $2 \mathrm{mM}$ GDP for FucT.

\subsection{SDS-PAGE and in-gel digestion}

Active fractions by enzymatic assay were pooled and subjected to trichloroacetic acid (TCA) precipitation. The resulting pellets were redissolved in $20 \mu \mathrm{L}$ SDS-PAGE sample buffer and boiled for $5 \mathrm{~min}$. SDS-PAGE 1-D gel was run until the dye front passed the stacking gel and then stained by EZblue protein staining kit (Sigma). Stained region was excised for standard in-gel digestion. Briefly, the gel pieces were subjected to reduction with $55 \mathrm{mM}$ dithioerythreitol in $25 \mathrm{mM}$ ammonium bicarbonate, $\mathrm{pH} 8.5$, at $37^{\circ} \mathrm{C}$ for $1 \mathrm{~h}$, and subsequently alkylated with $100 \mathrm{mM}$ iodoacetamide in $25 \mathrm{mM}$ ammonium bicarbonate, $\mathrm{pH}$ 8.5, at room temperature for $1 \mathrm{~h}$. The gel pieces were then washed twice with $50 \%$ $\mathrm{ACN}$ in $25 \mathrm{mM}$ ammonium bicarbonate, $\mathrm{pH} 8.5$ for $15 \mathrm{~min}$ each time, dehydrated with ACN for 5 min, dried, and finally rehydrated with $25 \mathrm{mM}$ ammonium bicarbonate, $\mathrm{pH} 8.5$, containing a total of $0.1 \mu \mathrm{g}$ of sequencing grade, modified trypsin (Promega) for $16 \mathrm{~h}$ at $37^{\circ} \mathrm{C}$. Following digestion, tryptic peptides were extracted twice by $50 \%$ ACN containing $5 \%$ TFA for $3 \mathrm{~min}$ with $10 \mathrm{~s}$ interval sonication. The extracted solutions were pooled and dried.

\subsection{LC-MS/MS and protein ID}

LC-nano-ESI-MS/MS analysis were performed on an integrated nano-LC-MS/MS system (Micromass) with an autosampler, a stream select module configured for precolumn plus analytical capillary column, and a Micromass Q-Tof Ultima API mass spectrometer fitted with nano-LC sprayer, operated under MassLynx 4.2 control. Injected samples were first trapped and desalted isocratically on a homemade precolumn (Nuclesil C18, $5 \mu \mathrm{m}$ particle size, $100 \AA$ pore size) for 4 min with $0.1 \%$ formic acid delivered at $15 \mu \mathrm{L} / \mathrm{min}$ after which the peptides were eluted off from the precolumn and separated on an analytical C18 capillary column (packed with the same material as the precolumn) connected in-line to the mass spectrometer, for a $60 \mathrm{~min}$ gradient of $0-80 \% \mathrm{ACN}$ in $0.1 \%$ formic acid, at $\sim 300 \mathrm{~nL} / \mathrm{min}$.

After data acquisition, the individual MS/MS spectra within a single LC run were combined, smoothed, deisotoped using the Micromass ProteinLynx ${ }^{\mathrm{TM}}$ Global Server 
(PGS) 2.0 and output as a single peak list (.pkl) file. The peak list files were used to query the Swiss-Prot database for human proteins using the MASCOT program (Version: 1.9.05). Enzyme digestion was set to trypsin allowing one miss cleavage. Carboxyamidomethylation on cysteine and oxidation on methionine were set as variable modification. The precursor ion and fragment ion tolerance were set to 0.25 Da. For each sample, an exclusion list, based on protein ID result, was generated for a second LC-MS/MS run to exclude previous found peptides. The two separate peak list files from the same sample thus obtained were manually combined into one single.pkl file for database searching. To minimize false positive, each individual peptide ion with a MASCOT score lower than the significant level was removed (scores of 31 for $p<0.05$ ).

\section{Results and discussion}

The unusual occurrence of extended type 1 chain on the GSLs of colo205 has been attributed to an abnormally high expression level of $\beta 3 \mathrm{GalT} 5$ among the candidate $\beta 3 \mathrm{GalTs}$, as demonstrated at the mRNA transcript level [18]. Consequently, we would expect $\beta 3$ GalT5 to be readily identified among the glycosyltransferases in a corresponding proteomic analysis. This would serve as a positive control against detecting any other targeted Golgi glycosyltransferases of interest. A major aim of current investigation was to establish which of the $\alpha 3 / 4$ FucTs among FucT3-7, and FucT9, can be identified at protein expression level, in comparison against their reported transcript level [19, 20]. However, initial attempt using either microsome or Golgi preparation failed to identify any of these glycosyltransferases through standard shotgun proteomic analysis. This is consistent with current limitations in the sensitivity and selectivity of detection without target enrichment. Thus, short of readily available $\mathrm{mAb}$, a robust enzymatic assays which can resolve specifically the targeted $\beta 3 \mathrm{GalT}$ and $\alpha 3 / 4 \mathrm{FucT}$ activities were first developed and applied to monitor the subfractionation procedures for a more targeted approach.

Instead of conventional radioisotope-based assay which is simple but lack of structure specific information, fluorescence tagged PA-sugar chains were used as acceptor substrates for nonradioactivity-based enzymatic activity assays. This method confers the advantage of easy monitoring and separation of products by HPLC [21], which can be further subjected to facile MS and MS/MS analysis to ascertain the identity of the synthesized products. In particular, since both $\beta 3 \mathrm{GalT}$ and $\beta 4 \mathrm{GalT}$ compete for the same substrate and, likewise, all $\alpha 3 / 4 \mathrm{FucT}$ s share the same lacto-series acceptor substrates with $\alpha 2 \mathrm{FucT}$, resolving the structural isomers of the enzymatic products are required. As shown in Fig. 1, the
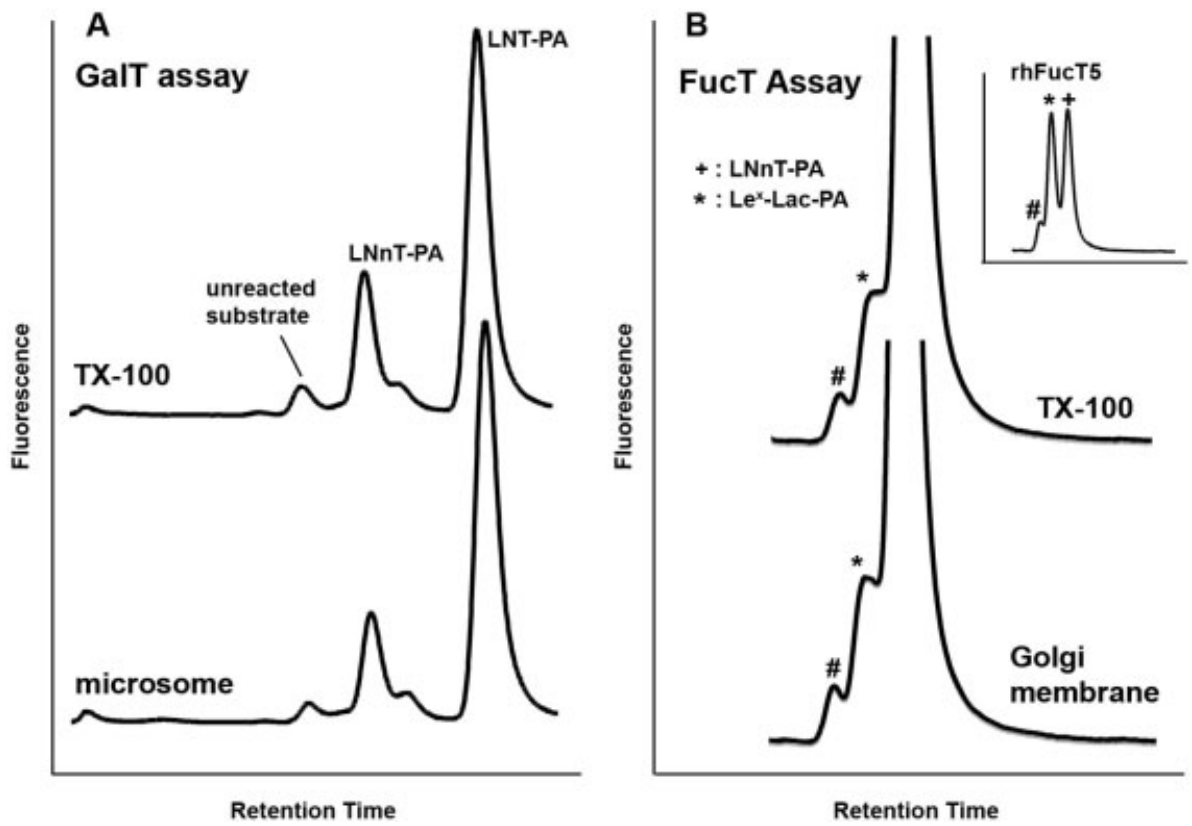

Figure 1. GalT and FucT enrichment and activity assay based on PA-glycans as acceptor substrates. Enzyme reaction products were separated by RP-HPLC and identified by fluorescent detector, in reference to the retention times of authentic standards. Quantitative assessment of individual products relative to unreacted substrates was afforded by integration of peak area. $\beta$ GalT activity is shown to be efficiently solubilized from the pelleted microsome fraction by $1 \%$ TX -100 , by comparing the product yield afforded by the microsome (lower trace) relative to that of the TX-100 extracts (upper trace). Likewise, $\alpha 3 / 4$ FucT activity was efficiently solubilized by $1 \%$ TX-100 (upper trace) from the Golgi membrane preparation (lower trace). The retention time of the $\alpha 3$-fucosylated product, Lewis $\times\left(\mathrm{Le}^{\mathrm{x}}\right)$-Lac-PA, was determined by using recombinant human FucT5 (rhFucT5, Calbiochem) as the enzyme source (see inset). The peak denoted by \# indicates contaminant substance also found in the original PA-sugar substrate stock. 


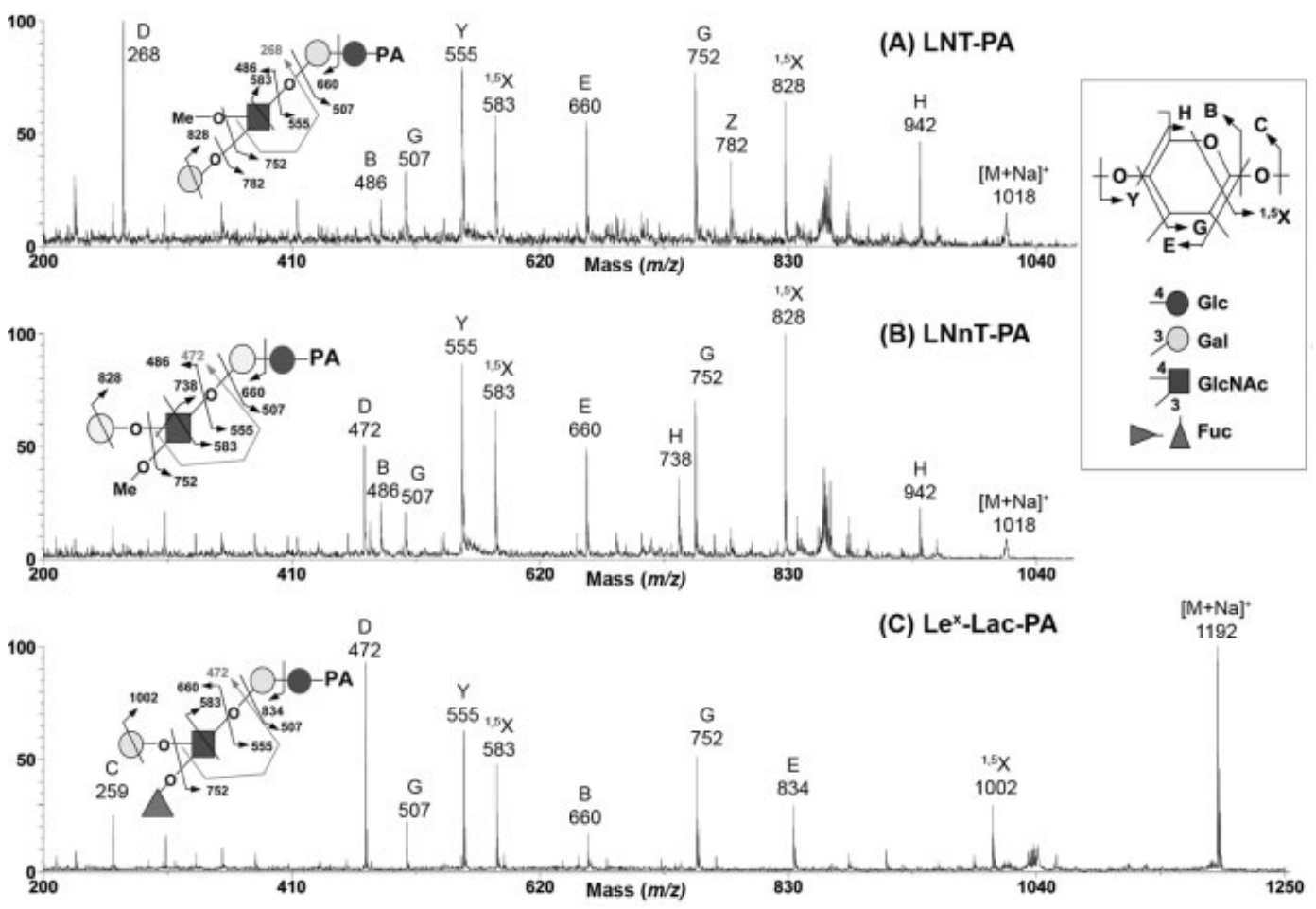

Figure 2. MALDI TOF/TOF CID-MS/MS sequencing of the GalT and FucT reaction products to distinguish between LNT-PA (A) and LNnT-PA (B), and to confirm the $\alpha 3$-fucosylation position at the GIcNAc of LNnT-PA, corresponding to Le $\mathrm{e}^{\mathrm{x}}$-Lac-PA (C). Critical fragment ions afforded by the permethyl derivatives [17] are the $D$ ions at $m / z 268$ and 472, which is specific to type 1 and 2 chain, respectively, as illustrated. For Le ${ }^{\mathrm{x}}$-Lac-PA, the type 2 chain specific $\mathrm{D}$ ion at $\mathrm{m} / \mathrm{z} 472$ confirms the $\alpha 3$-fucosylation at GlcNAc, whereas the absence of $\alpha 2$-FucT product was indicated by lack of both $\mathrm{C}$ and $\mathrm{D}$ ions at $\mathrm{m} / \mathrm{z} 433$ (Fuc-Gal-OH) and 646 (Fuc-Gal- $\Delta$ GlcNAc-OH, where $\Delta$ indicates an unsaturated bond due to elimination of 3-substituent). Symbol keys used and nomenclature of cleavage ions as annotated are further illustrated schematically (inset) and have been described in detail before [17].

unreacted GlcNAc $\beta 1$-Lac-PA substrate, Gal $\beta 1-3 G l c N A c \beta 1$ Lac-PA (type 1 chain, lactotetraose (LNT)-PA) and Gal $\beta 1$ 4 GlcNAc $\beta 1$-Lac-PA (type 2 chain, LNnT-PA) could be separated by RP-HPLC chromatography, identified first by referring to the elution time of available standard PA-glycan chains and then confirmed by MS/MS analysis (Fig. 2). For FucT assay, since all FucTs known to show $\alpha 4$-fucosylation activity against type 1 chain can also $\alpha 3$-fucosylate type 2 chain, the acceptor LNnT-PA was used as a general substrate to monitor all $\alpha 3 / 4$ FucT activities. The fucosylated product can be resolved from the precursor and the exact fucosylated position can be similarly distinguished based on both elution time and MS/MS analysis (Figs. 1 and 2).

Based on these PA-glycan assays, significant GalT and FucT activities were detected for the total lysates, as well as the microsome or Golgi fractions. As expected for Colo205, $\beta 3 \mathrm{GalT}$ activity which is normally very weak in other cell lines (data not shown) was prominently detected along with $\beta 4 \mathrm{GalT}$ activity. More importantly, we showed that the enzyme activities could be efficiently extracted from the membrane by $1 \%$ TX-100 (Fig. 1). This is critical since the targeted glycosyltransferases are type II membrane proteins, which often require detergent solubilization to improve recovery and prevent aggregation. Monitoring against the activities of $\beta 3 / 4 \mathrm{GalTs}$, a significant portion of the solubilized activities was shown to be retained on a UDP-affinity column and eluted by 2 mM UDP, after extensive wash (Fig. 3), which therefore suggested that it may constitute an effective affinity enrichment step. The continued use of detergent and glycerol in the eluting buffer served to stabilize the membrane proteins but nevertheless rendered the sample not conducive to the following in solution digestion and direct LC-MS/MS analysis. To get around this problem, and to prepare the enzyme active fractions for proteomic identification, the eluted sample was desalted and concentrated by TCA precipitation. The resulting pellet was then subjected to 1-D SDS-PAGE but the electrophoretic running was stopped immediately after the dye front migrated through the stacking gel. This method allows total proteins to be stacked as a band in the 1-D gel for subsequent in-gel digestion and LC-MS/MS analysis.

As a result, both $\beta 3$ GalT5 and $\beta 4 \mathrm{GalT} 1$ were positively identified by three and two matched peptides, respectively (Table 1). However, from a total of 95 proteins identified, only seven proteins may be classified as ER/Golgi proteins (data not shown). Despite the use of microsome preparation and 
Table 1. GalTs identified

\begin{tabular}{|c|c|c|c|}
\hline Protein name & Swiss-Prot ID & MASCOT score ${ }^{a)}$ & Sequence identified ${ }^{\text {b) }}$ \\
\hline$\beta 3$ GalT5 & P15291 & 162 & $\begin{array}{l}\text { FFTGFLK (34) } \\
\text { TFFLLGTTSSAAETK (57) } \\
\text { TLLDYWOALENSR (71) }\end{array}$ \\
\hline$\beta 4 \mathrm{GaIT} 1$ & Q9Y2C3 & 164 & $\begin{array}{l}\text { ETM*LSDGLNSLTYOVLDVOR (48) } \\
\text { LPQLVGVSTPLOGGSNSAAAIGQSSGELR (116) }\end{array}$ \\
\hline
\end{tabular}

a) MASCOT score of individual peptide is listed in parenthesis after the assigned peptide sequence.

b) $\mathrm{M}^{*}$ indicates oxidation at methionine.

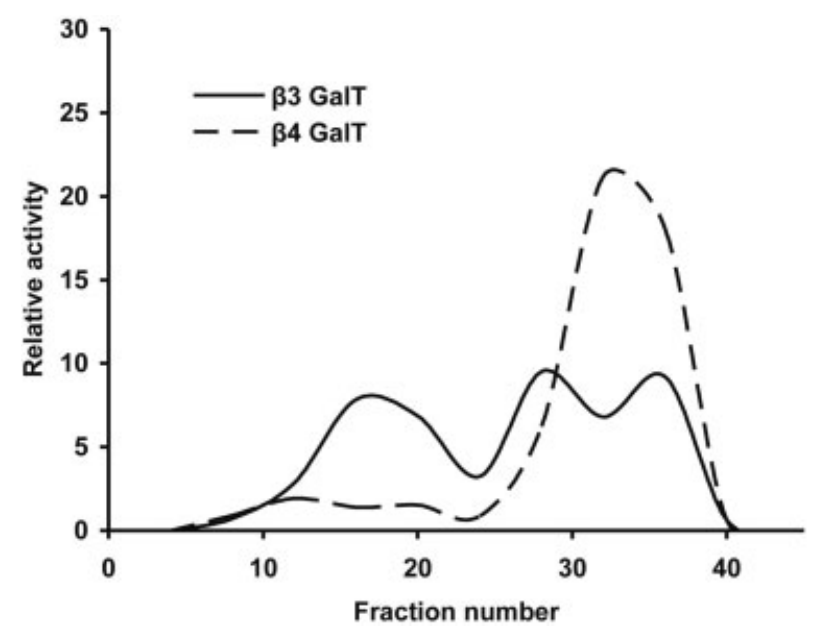

Figure 3. UDP-affinity enrichment of $\beta 3 \mathrm{GalT}$ and $\beta 4 \mathrm{GalT}$ activities extracted from colo205 microsome preparation. Collected fractions were subjected to $\beta$ GalT assay to monitor the distribution of $\beta 3 \mathrm{GalT}$ and $\beta 4 \mathrm{GalT}$ activities. Two-thirds of $\beta 3 \mathrm{GalT}$ was retained whereas $\beta 4$ GalT activity was fully retained by the UDP-affinity column. The bound fractions (\#30-38) were subjected to SDSPAGE and proteomic identification. Although $\beta 3 G$ alT activity can be found in the flow through (\#12-20), it cannot be efficiently identified in the presence of many other copurifying proteins.

the affinity step, it remains difficult to minimize the copurified cytosolic or nucleus proteins, including many DNA/ RNA binding proteins that presumably exhibited certain degree of affinity against UDP. To further improve the efficiency of enrichment, a Golgi preparation step was further incorporated into this overall protocol for subsequent proteomic analysis of the FucTs, substituting UDP with a GDPaffinity column. Thus, Golgi membrane was separated from postnuclear fraction by zonal ultracentrifugation with step sucrose gradient. The activities of the targeted $\alpha 3 / 4$ FucTs were shown to colocalize with the trans-Golgi marker, $\beta 4$ GalT activity (Fig. 4), and the enriched Golgi fractions were pelleted by ultracentrifugation and subjected to similar TX100 extraction.

Before sample loading onto the GDP-affinity column, the TX-100 content was adjusted to the condition for maximum binding ability, which is often also the condition for best

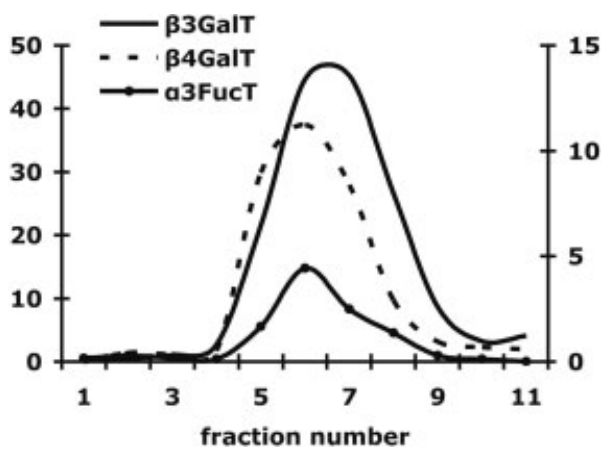

Figure 4. Subcellular fractionation of Colo205 membrane was conducted by a step sucrose gradient from 15 to $55 \%$. Fractions were collected from top to bottom of gradient. $\beta 4$ GalT activity assay was conducted to locate the distribution of Golgi membrane. Distribution of $\beta 3 \mathrm{GalT}$ and $\alpha 3 \mathrm{FucT}$ was also evaluated and found to colocalize with the $\beta 4 \mathrm{GalT}$ activity. Left $Y$-axis indicates the relative activity of $\beta 3 \mathrm{GalT}$ whereas right $Y$-axis indicates the relative activities of $\beta 4 \mathrm{GaIT}$ and $\alpha 3 \mathrm{FucT}$.

enzyme activity. To remove nonspecific binding, $3 \times$ volume of binding buffer and $0.5 \mathrm{M} \mathrm{NaCl}$ in binding buffer were used consecutively to wash the GDP-affinity resin. GDP-affinity bound fraction was then eluted by $2 \mathrm{mM}$ GDP and subjected to $\alpha 3 / 4 \mathrm{FucT}$ assay to evaluate the distribution of $\alpha 3 / 4$ FucT activity. As shown in Fig. 5, the $\alpha 3 / 4$ FucT activity was well retained by the GDP-affinity resin. As in the treatment of $\beta$ GalTs, the detergent carrying eluates were TCA precipitated, similarly run into 1-D gel as a single focused band, followed by in-gel digestion and LC-MS/MS shotgun proteomic analysis of the extracted peptides.

Three FucTs, FucT3, FucT6, and OFUT1, were positively identified from the $\alpha 3 / 4 \mathrm{FucT}$ active fraction (Table 2). In addition to three common peptide matches, the highly homologous FucT3 and FucT6 [22] are distinguished respectively by one and two additional unique peptide matches of sufficiently good MASCOT scores. In vitro studies have demonstrated that only FucT3 and FucT5 possess $\alpha 1$,4FucT activity to fucosylate type 1 chain. Since the expression of FucT5 is undetectable at both mRNA transcript level $[19,20]$ and by current proteomic analysis, it could therefore be concluded that FucT3 is the only enzyme responsible for 
Table 2. FucTs identified

\begin{tabular}{|c|c|c|c|}
\hline Protein name & Swiss-Prot ID & MASCOT score ${ }^{a)}$ & Sequence identified ${ }^{\text {b) }}$ \\
\hline FucT6 & P51993 & 231 & $\begin{array}{l}\text { SFSWALAFC*K (41) } \\
\text { EVMYNPSAQLPR (57) } \\
\text { YLOELDKDHAR (51) } \\
\text { NALEAWAVPVVLGPSR (48) } \\
\text { FLPPDAFIHVDDFQSPK (34) }\end{array}$ \\
\hline FucT3 & P21217 & 187 & $\begin{array}{l}\text { SFSWALDFC*K (54) } \\
\text { YLQELDKDHAR (51) } \\
\text { NALEAWAVPVVLGPSR (48) } \\
\text { FLPPDAFIHVDDFOSPK (34) }\end{array}$ \\
\hline OFUT1 & Q9H488 & 71 & VISLEDFMEK (71) \\
\hline
\end{tabular}

a) MASCOT score of individual peptide is listed in parenthesis after the assigned peptide sequence.

b) Sequences underlined are common to FucT3 and FucT6; C* indicates carboxyamidomethylation at cysteine.

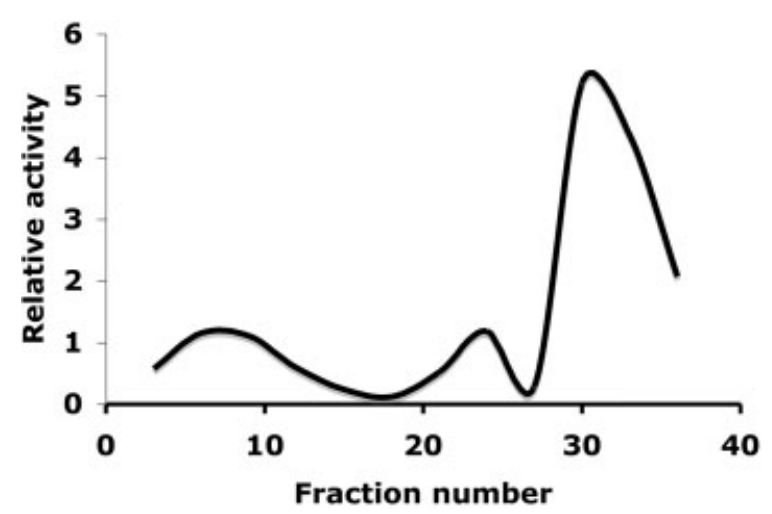

Figure 5. GDP-affinity enrichment of $\alpha 3 / 4 \mathrm{FucT}$ activity extracted from Colo205 Golgi preparation. Collected fractions were subjected to $\alpha 3$ FucT assay to locate the distribution of $\alpha 3$ FucT activity. $\alpha 3 / 4 F u c T$ activity was well retained by the GDP-affinity column. The bound fractions (\#34-38) were subjected to SDS-PAGE and proteomic ID.

synthesizing Le $\mathrm{a}^{\mathrm{a}}$ and (sialyl) Lewis a, (sialyl)Gal $\beta 1$-(Fuc $\alpha 1$ 4) 3 GlcNAc $\beta$ - (s) Le a $^{\mathrm{a}}$ in Colo205. On the other hand, FucT3 and FucT6 could both account for $\alpha 3$-fucosylation of type 2 chain in Colo205, among the many known $\alpha 3$ FucTs. The proteomic results are in fact in good correlation with previous findings, which demonstrated that Colo205 cells express high levels of FucT3 and FucT6 transcripts but relatively low level of other $\alpha 3 / 4$ FucTs [23], like most other cells of epithelial origin [24].

An interesting result is the identification of OFUT1 in the $\alpha 3 / 4 F u c T$ active fractions, which suggests an equally high binding affinity to GDP resin. Recent studies have implicated OFUT1 as an ER lumenal protein instead of Golgi resident membrane protein [25]. Our proteomic studies using microsome fraction for GDP-affinity enrichment has always led to identification of OFUT1 with higher sequence coverage (data not shown), instead of only one matched peptide when using the Golgi preparation, whereas the reverse is true for FucT3 and FucT6. This not only suggests a better enrichment efficiency of $\alpha 3 / 4$ FucTs by Golgi preparation but also consistent with OFUT1 being primarily an ER protein. The better known biological function of OFUT1 is to catalyze protein $O$-fucosylation of the conserved EGF domain in a number of proteins involved in Notch signaling [26-29]. OFUT1 was shown to be highly expressed only in heart, brain, placenta, lung, liver, skeletal muscle, kidney, and pancreas [28]. Our proteomic identification of OFUT1 suggests a relatively high protein expression level in Colo205 cells but the biological implication remains to be elucidated.

Aside from keratins, a total of 52 proteins were confidently identified in the $\alpha 3 / 4 \mathrm{FucT}$ active fraction (see Table in Supporting Information) according to the criteria described in Section 2. Among these, those formally classified as ER/Golgi proteins according to Swiss-Prot annotation account for $31 \%$ of total, suggesting a good enrichment of ER/Golgi membrane comparable to that reported in the literature [12, 14]. However, only five proteins have been shown to interact with GDP/GTP and 16 proteins with ATP/UDP, based on the Swiss-Prot annotation. It thus appears that a significant portion of coeluting proteins was not specifically enriched by affinity to GDP. More extensive wash and/or preadsorption with the resins would conceivably reduce further the nonspecific interactions but unlikely to have yielded only the desirable GDP-binding proteins.

\section{Concluding remarks}

Identification of glycosyltransferases represents one of the most daunting undertakings in current proteomics. The glycosyltransferases are not only of low abundance but are also Golgi resident membrane proteins. A range of technical issues therefore need to be considered including extraction, subcellular fractionation, detergent solubilization, affinity 
enrichment, and efficient digestion, all of which must lead to final compatibility with MS analysis without incurring lengthy buffer exchange steps of poor recovery. In the absence of readily available antibodies against the endogenous enzymes or activity-based probe of sufficient reactivity and specificity, we have shown that both Golgi and GDP-affinity enrichment, coupled with semigel-based approach, are the necessary minimal workflow for successful identification by shotgun proteomics. Our proteomic results, as exemplified here with applications to Colo205 cells, are largely consistent with mapping at the mRNA transcript level in so far as revealing the major $\beta 3 \mathrm{GalT}$ and $\alpha 3 / 4 \mathrm{FucT}$ activities. However, only at protein level, through further optimization and scale up, would it be possible to contemplate tackling potential PTMs.

We are grateful to Professor Naoyuki Taniguchi and Dr. Tomohiko Taguchi (Department of Biochemistry, Osaka University Graduate School of Medicine) for their technical advice to CHL in the biochemical handling of glycosyltransferases and PAsugar activity assay. This work is supported by an Academia Sinica Program Project Grant. Proteomic analysis was performed at the National Core Facilities for Proteomics (NSC grant 953112-B-001-014), located at the Institute of Biological Chemistry, Academia Sinica.

The authors have declared no conflict of interest.

\section{References}

[1] Sadler, J. E., Beyer, T. A., Oppenheimer, C. L., Paulson, J. C. et al., Purification of mammalian glycosyltransferases. Meth. Enzymol. 1982, 83, 458-514.

[2] Paulson, J. C., Colley, K. J., Glycosyltransferases. Structure, localization, and control of cell type-specific glycosylation. $J$. Biol. Chem. 1989, 264, 17615-17618.

[3] Narimatsu, H., Sinha, S., Brew, K., Okayama, H., Qasba, P. K., Cloning and sequencing of cDNA of bovine $\mathrm{N}$-acetylglucosamine (beta 1-4)galactosyltransferase. Proc. Natl. Acad. Sci. USA 1986, 83, 4720-4724.

[4] Narimatsu, H., Human glycogene cloning: Focus on beta 3glycosyltransferase and beta 4-glycosyltransferase families. Curr. Opin. Struct. Biol. 2006, 16, 567-575.

[5] Narimatsu, H., Construction of a human glycogene library and comprehensive functional analysis. Glycoconj. J. 2004, $21,17-24$.

[6] Stults, J. T., Arnott, D., Proteomics. Meth. Enzymol. 2005, 402, 245-289.

[7] Hathaway, H. J., Evans, S. C., Dubois, D. H., Foote, C. I. et al., Mutational analysis of the cytoplasmic domain of beta1,4galactosyltransferase I: Influence of phosphorylation on cell surface expression. J. Cell Sci. 2003, 116, 4319-4330.

[8] de Graffenried, C. L., Bertozzi, C. R., The roles of enzyme localisation and complex formation in glycan assembly within the Golgi apparatus. Curr. Opin. Cell Biol. 2004, 16, 356-363.
[9] Yates, J. R., III, Gilchrist, A., Howell, K. E., Bergeron, J. J., Proteomics of organelles and large cellular structures. Nat. Rev. Mol. Cell Biol. 2005, 6, 702-714.

[10] Mogelsvang, S., Howell, K. E., Global approaches to study Golgi function. Curr. Opin. Cell Biol. 2006, 18, 438-443.

[11] Bell, A. W., Ward, M. A., Blackstock, W. P., Freeman, H. N. et al., Proteomics characterization of abundant Golgi membrane proteins. J. Biol. Chem. 2001, 276, 5152-5165.

[12] Wu, C. C., MacCoss, M. J., Mardones, G., Finnigan, C. et al., Organellar proteomics reveals Golgi arginine dimethylation. Mol. Biol. Cell 2004, 15, 2907-2919.

[13] Takatalo, M. S., Kouvonen, P., Corthals, G., Nyman, T. A., Ronnholm, R. H., Identification of new Golgi complex specific proteins by direct organelle proteomic analysis. Proteomics 2006, 6, 3502-3508.

[14] Wu, C. C., Yates, J. R., III, Neville, M. C., Howell, K. E., Proteomic analysis of two functional states of the Golgi complex in mammary epithelial cells. Traffic 2000, 1, 769-782.

[15] Stroud, M. R., Levery, S. B., Nudelman, E. D., Salyan, M. E. et al., Extended type 1 chain glycosphingolipids: Dimeric Lea (III4V4Fuc2Lc6) as human tumor-associated antigen. J. Biol. Chem. 1991, 266, 8439-8446.

[16] Holmes, E. H., Characterization and membrane organization of beta 1-3- and beta 1-4-galactosyltransferases from human colonic adenocarcinoma cell lines Colo 205 and SW403: Basis for preferential synthesis of type 1 chain lacto-series carbohydrate structures. Arch. Biochem. Biophys. 1989, 270, 630-646.

[17] Yu, S. Y., Wu, S. W., Khoo, K. H., Distinctive characteristics of MALDI-Q/TOF and TOF/TOF tandem mass spectrometry for sequencing of permethylated complex type N-glycans. Glycoconj. J. 2006, 23, 355-369.

[18] Isshiki, S., Togayachi, A., K, T., Nishihara, S. et al., Cloning udo, expression, and characterization of a novel UDP-galactose: Beta- $\mathrm{N}$-acetylglucosamine beta1,3-galactosyltransferase (beta3Gal-T5) responsible for synthesis of type 1 chain in colorectal and pancreatic epithelia and tumor cells derived therefrom. J. Biol. Chem. 1999, 274, 12499-12507.

[19] Sasaki, K., Kurata, K., Funayama, K., Nagata, M. et al., Expression cloning of a novel alpha 1,3-fucosyltransferase that is involved in biosynthesis of the sialyl Lewis $x$ carbohydrate determinants in leukocytes. J. Biol. Chem. 1994, 269, 14730-14737.

[20] Kaneko, M., Kudo, T., Iwasaki, H., Ikehara, Y. et al., Alpha1,3fucosyltransferase IX (Fuc-TIX) is very highly conserved between human and mouse; molecular cloning, characterization and tissue distribution of human Fuc-TIX. FEBS Lett. 1999, 452, 237-242.

[21] Taniguchi, N., Nishikawa, A., Fujii, S., Gu, J. G., Glycosyltransferase assays using pyridylaminated acceptors: $\mathrm{N}$ acetylglucosaminyltransferase III, IV, and V. Meth. Enzymol. 1989, 179, 397-408.

[22] de Vries, T., Knegtel, R. M., Holmes, E. H., Macher, B. A., Fucosyltransferases: Structure/function studies. Glycobiology 2001, 11, 119R-128R.

[23] Hiller, K. M., Mayben, J. P., Bendt, K. M., Manousos, G. A. et al., Transfection of alpha(1,3)fucosyltransferase antisense sequences impairs the proliferative and tumorigenic ability of human colon carcinoma cells. Mol. Carcinog. 2000, 27, 280-288. 
[24] Kannagi, R., Izawa, M., Koike, T., Miyazaki, K., Kimura, N., Carbohydrate-mediated cell adhesion in cancer metastasis and angiogenesis. Cancer Sci. 2004, 95, 377-384.

[25] Luo, Y., Haltiwanger, R. S., O-fucosylation of notch occurs in the endoplasmic reticulum. J. Biol. Chem. 2005, 280, 1128911294.

[26] Wang, Y., Lee, G. F., Kelley, R. F., Spellman, M. W., Identification of a GDP-L-fucose: Polypeptide fucosyltransferase and enzymatic addition of O-linked fucose to EGF domains. Glycobiology 1996, 6, 837-842.
[27] Wang, Y., Spellman, M. W., Purification and characterization of a GDP-fucose: Polypeptide fucosyltransferase from Chinese hamster ovary cells. J. Biol. Chem. 1998, 273, 81128118.

[28] Wang, Y., Shao, L., Shi, S., Harris, R. J. et al., Modification of epidermal growth factor-like repeats with O-fucose. Molecular cloning and expression of a novel GDP-fucose protein O-fucosyltransferase. J. Biol. Chem. 2001, 276, 40338-40345.

[29] Haines, N., Irvine, K. D., Glycosylation regulates Notch signalling. Nat. Rev. Mol. Cell Biol. 2003, 4, 786-797. 